\title{
A CONVERGENT EXPRESSION FOR THE PARTITION FUNCTION OF A PARTIALLY IONIZED HYDROGEN PLASMA
}

\author{
by R. DEKEYSER*) \\ Instituut voor Theoretische Fysica, Rijksuniversiteit, Utrecht
}

\section{Synopsis}

The partition function of a hydrogen plasma is derived, partly classically and partly quantum-mechanically, avoiding in this way at the same time the close distance and the high orbit divergences. Our result is exact in the small density and infinite temperature limit, but can be considered as a good approximation also for conditions, where the ionization is incomplete. From this a Saha equation is derived, which contains an effective lowering of the ionization potential. An expression is obtained for the pressure, which is not simply the sum of the known pressures of the neutral and ionized parts, but has a correction from the transitions between these parts.

Introduction. As is well known, the partition function of a hydrogen gas diverges, due to the infinite number of energy levels, corresponding to high principal quantum numbers. Herzfeld ${ }^{1}$ ) was the first to investigate this problem, which he tried to solve by a cutoff at a certain maximum Bohr radius. The interpretation of this maximum radius, however, was not clear. Instead of this cutoff, Fowler ${ }^{2}$ ) proposed a continuous decrease of the occupation probabilities of the energy states, and $\mathrm{Fermi}^{3}$ ) derived a concrete expression for this decrease. Variations on these methods were presented by Becker ${ }^{4}$ ), Planck ${ }^{5}$ ), Riewe and Rompeb). Recently, Larenz ${ }^{7}$ ) gave a solution by a rather heuristic junction between the weight functions for bound and free energy states.

An other problem is the non-existence of a classical hydrogen plasma: there are always divergences from small electron-proton distances, which must be treated quantummechanically. This difficulty is most often avoided by the assumption that the positive charges are smeared out. The question arises, however, if a correct quantummechanical treatment would not reveal important corrections to the results obtained by the smearing out.

In the present paper we try to solve both problems at the same time. The lowest bound energy stares are treated quantummechanically, with

*) Aangesteld navorser van het Belgisch Nationaal Fonds voor Wetenschappelijk Onderzoek. 
neglect of the plasma in which the atom is immersed. The higher energy states will be described classically and from a collective point of view. The boundary between these two descriptions is still arbitrary (between certain limits), and we have to prove that our results do not depend on it.

In the first section we describe our system, and we investigate the conditions under which our assumptions are valid. The second and third sections give the calculations of the partition function, and in section 4 we derive from it the Saha equation. In section 5 we prove that our expression for the partition function is independent of the definite choice for the boundary between our two descriptions; finally, as an application, we calculate the pressure of our gas.

$\S 1$. General ideas. For definiteness we consider a plasma consisting of $N$ electrons and $N$ protons, with masses $m_{e}$ and $m_{p}$ and charges $-e$ and $+e$. We introduce

$$
\begin{aligned}
& M=m_{e}+m_{p} \\
& m=\frac{m_{e} m_{p}}{M}
\end{aligned}
$$

We suppose that these particles are contained in a volume $\mathrm{V}$, and denote $\rho=N / V$.

There will be for our particles a possibility to form bound pairs. It is obvious to describe these pairs quantummechanically by omitting the influence of the rest of the plasma; this must be possible if the plasma is sufficiently dilute. The possible internal energies of these pairs will then approximately be the energies $E_{n}$ of the hydrogen atom, corresponding to the principal quantum number $n$ with degeneracy $n^{2}$. We do not take account of the spin degeneracy.

We shall, however, only maintain this quantummechanical description up to a certain energy limit $E_{\nu}$ (which is negative). For every other electronproton pair, there still remain two possibilities. If the distance $r$ between them is less than $\left|e^{2} / E_{\boldsymbol{v}}\right|$, their relative momentum $p$ must be greater than $\left[2 m\left(e^{2} / r+E_{v}\right)\right]^{ \pm}$, in order to satisfy the condition that their internal energy be greater than $E_{\nu}$. If the distance is greater than $\left|e^{2} / E_{\nu}\right|$, there is no lower limit for that momentum. Each of these possibilities will be described classically.

In order to be able to make such a cut between a classical and a quantummechanical description, there are some conditions to be fulfilled:

$a$. The energy states above the limit must lie close enough together to allow us to consider them as an "almost continuous spectrum". This means

$$
v \gg 1
$$


$b$. The quantummechanical energies of the hydrogen atom may not considerably be altered by the presence of the other plasma particles. This will only be satisfied if the radius of the highest quantized orbit is much smaller than the mean interparticle distance:

$$
\left\langle r_{v}\right\rangle=\left|\frac{e^{2}}{2 E_{v}}\right|=\frac{v^{2} \hbar^{2}}{m e^{2}} \ll\left(\frac{3}{4 \pi \rho}\right)^{t}=r_{0} .
$$

We impose one further condition, not so much for fundamental reasons as for convenience:

c. $k_{B} T \gg\left|E_{\eta}\right|$, where $k_{B}$ is Boltzmann's constant and $T$ the temperature. This provides us with a small parameter $\beta\left|E_{\nu}\right|\left(\beta=1 / k_{B} T\right)$ for series developments of otherwise hardly manageable functions:

The situation, which permits a perfect combination of conditions $a$ and $b$ is the infinitely dilute plasma. If we want to generalize our calculations to plasmas with densities up to $10^{16} \mathrm{~cm}^{-3}$, we shall have to content ourselves with $v \simeq 5-10$. Condition $c$ means that we have a high temperature description.

In the following table, we propose acceptable values of $v$, corresponding to several hydrogen plasma densities. In each case we indicate $T_{\min }$, the minimum value of the temperature that satisfies condition $c$.

TABLE 1

\begin{tabular}{|c|c|c|c|c|}
\hline$\rho\left(\mathrm{cm}^{-3}\right)$ & $r_{0}(\mathrm{~cm})$ & possible $\nu$ & $r_{y}(\mathrm{~cm})$ & $T_{\mathrm{min}}\left({ }^{\circ} \mathrm{K}\right)$ \\
\hline $10^{6}$ & $6 \cdot 10^{-3}$ & 100 & $5 \cdot 10^{-5}$ & 50 \\
$10^{9}$ & $6 \cdot 10^{-4}$ & 40 & $10^{-5}$ & 300 \\
$10^{12}$ & $6 \cdot 10^{-5}$ & 30 & $5 \cdot 10^{-6}$ & 500 \\
$10^{15}$ & $6 \cdot 10^{-6}$ & 10 & $5 \cdot 10^{-7}$ & $5 \cdot 10^{3}$ \\
$10^{18}$ & $6 \cdot 10^{-7}$ & 5 & $10^{-7}$ & $2 \cdot 10^{4}$ \\
\hline
\end{tabular}

We can also apply our results to low temperature media as e.g. the ionosphere by weakening condition $b$. We must then suppose that neutral atoms do not disturb the electron orbits, so that we have to take account only of the density of ionized particles in condition $b$.

To have a consistent description, we must further note that the quantummechanical form of the partition function of a system of $t$ particles with energy levels $E$ and degeneracy $g(E)$

$$
\sum_{E} g(E) \exp (-\beta E)
$$

corresponds with the classical form

$$
h^{-3 t} \int \ldots \int \mathrm{d} \boldsymbol{r}_{1} \ldots \mathrm{d} \boldsymbol{r}_{t} \mathrm{~d} \boldsymbol{p}_{1} \ldots \mathrm{d} \boldsymbol{p}_{t} \exp \left[-\beta E\left(\boldsymbol{r}_{1}, \ldots, \boldsymbol{r}_{t} ; \boldsymbol{p}_{1}, \ldots, \boldsymbol{p}_{t}\right)\right]
$$

§2. The different parts of the partition function. Let us suppose that we have between our particles $N_{n}$ electron-proton pairs in the $n$-th bound state 
with internal energy $E_{n}(n=1,2, \ldots, v)$ and $N^{\prime}$ pairs with a relative energy greater than $E_{p}$ but with a relative distance less than $\left|e^{2} / E_{p}\right|$. We further denote

$$
N^{\prime \prime}=N-N^{\prime}-N_{1}-N_{2}-\ldots-N_{v}=N-N^{0} .
$$

The partition function of our plasma will then be the sum of the contributions from all possible divisions of our $2 N$ particles in the $N_{n}$ and $N^{\prime}$ pairs and the $2 N^{\prime \prime}$ free particles. Let us write it as follows:

$$
Z=\sum_{\left\{N_{n}\right\}, N^{\prime}, N^{\prime}} \frac{N ! N !}{N_{1} ! \ldots N_{\nu} ! \overline{N^{\prime}} ! N^{\prime \prime} ! N^{\prime \prime} !} \prod_{n=1}^{\nu} Z_{n}^{N_{n}} \cdot Z^{\prime} N^{\prime} \cdot Z^{\prime \prime},
$$

and we shall try to calculate each of the unknown factors. The factorials arise from the number of ways in which each division can be performed. We immediately note that a part of the summation can be carried out, so that

$$
Z=\sum_{N^{0}+N^{\prime \prime}=N} \frac{N ! N !}{N^{0} ! N^{\prime \prime} ! N^{\prime \prime} !}\left(Z^{\prime}+\sum_{n=1}^{v} Z_{n}\right)^{N^{0}} \cdot Z^{\prime \prime} .
$$

$Z_{n}$ is very simple to write down: it contains the degeneracy $n^{2}$ of the $n$-th hydrogen level, the probability factor $\exp \left(-\beta E_{n}\right)$ and a factor from the translational motion of the center of mass of the bound pair:

$$
Z_{n}=V \cdot\left(\frac{2 \pi M}{\beta h^{2}}\right)^{\frac{3}{2}} \cdot n^{2} \exp \left(-\beta E_{n}\right)
$$

$Z^{\prime}$ contains the same factor from the center of mass motion, but we describe the internal motion with the classical formula (2), taking account of the boundary limits for the distance and relative momentum. Here too we disregard the influence of the other plasma particles. From now on, we write

We then have

$$
-E_{\nu}=A \text {. }
$$

$$
\begin{aligned}
Z^{\prime} & =V\left(\frac{2 \pi M}{\beta h^{2}}\right)^{i} h^{-3} \int_{0}^{e^{2 / A}} 4 \pi r^{2} \mathrm{~d} r \int_{\sqrt{2 m\left\{\left(e^{2} / r\right)-A\right\}}}^{\infty} 4 \pi p^{2} \mathrm{~d} p \exp \left[-\beta\left(\frac{p^{2}}{2 m}-\frac{e^{2}}{r}\right)\right]= \\
& =V\left(\frac{m M}{\pi \beta \hbar^{4}}\right)^{\prime} \frac{e^{6}}{\pi} \int_{-A}^{\infty} \mathrm{d} E \mathrm{e}^{-\beta E} \int_{A}^{\infty} \mathrm{d} y \frac{\sqrt{E+y}}{y^{4}}=V\left(\frac{m M}{\pi \beta \hbar^{4}}\right)^{t} . \\
& \cdot \frac{e^{6}}{\pi} \int_{A}^{\infty} \mathrm{d} y y^{-4}\left\{\frac{1}{\beta} \sqrt{y-A} \mathrm{e}^{\beta A}+\frac{\sqrt{ } \pi}{2 \beta^{\mathbf{1}}}[1-\Phi(\sqrt{\beta(y-A)})] \mathrm{e}^{\beta \gamma}\right\}
\end{aligned}
$$


where $\Phi(x)$ is the error integral, defined as

$$
\Phi(x)=-\frac{2}{\sqrt{ } \pi} \int_{0}^{x} \mathrm{e}^{-t^{2}} \mathrm{dt} .
$$

The difficult part in these calculations is the integral over the error function. We made use of the relation

$$
\left.\int_{\boldsymbol{A}}^{\infty} \mathrm{d} y(1-\Phi(\sqrt{\beta(y-A}))\right] \mathrm{e}^{\alpha \gamma}=\frac{1}{\alpha}\left[\frac{\sqrt{ } \beta}{\sqrt{\beta-\alpha}}-1\right] \mathrm{e}^{\alpha A},
$$

valid for $\beta>\alpha$, which we integrated four times over $\alpha$. Part of the remaining calculations can be carried through exactly, the rest can be approximated as a series in the small parameter $A \beta$. We do not give the lengthy calculations; the result is

$$
\begin{aligned}
Z^{\prime} & =V\left(\frac{4 \pi^{2} m M}{\beta^{2} h^{4}}\right)^{\frac{3}{3}} \frac{\pi e^{6}}{A^{3}}\left[\frac{4}{3}+2 A \beta-\frac{\sqrt{ } \pi}{3}(A \beta)^{3}+2(A \beta)^{2}-\right. \\
& \left.-\sqrt{\pi}(A \beta)^{1}-\frac{2}{3}(A \beta)^{3} \log (A \beta)+\ldots\right] .
\end{aligned}
$$

$Z$ " can be written as an integral over the space and momentum variables of the $2 N^{\prime \prime}$ remaining particles. As there is no limit to be imposed on the momentum variables, we can immediately integrate over them, which yields a factor

$$
\left(\frac{2 \pi m_{e}}{\beta h^{2}}\right)^{3 N^{\prime \prime} / 2} \cdot\left(\frac{2 \pi m_{p}}{\beta h^{2}}\right)^{3 N^{\prime \prime} / 2}
$$

On the space variables, however, we have to impose the condition that none of the $N^{\prime \prime}$ electrons may come closer than a distance $e^{2} / A$ to each proton. In a certain sense we have to describe the interaction potential with a hard core (H.C.). The rest of $Z^{\prime \prime}$ is thus

$$
e^{G}=\int_{\text {H.C. }} \ldots \int \mathrm{d} \boldsymbol{x}_{1} \ldots \mathrm{d} \boldsymbol{x}_{N^{\prime \prime}} \mathrm{d} \boldsymbol{y}_{1} \ldots \mathrm{d} \boldsymbol{y}_{N^{*}} \exp \left[-\beta W\left(\boldsymbol{x}_{1}, \ldots, \boldsymbol{x}_{N^{*}} ; \boldsymbol{y}_{1}, \ldots, \boldsymbol{y}_{N^{*}}\right)\right],
$$

where we give coordinates $\boldsymbol{x}$ to the electrons and coordinates $\boldsymbol{y}$ to the protons. The function $W$ is the sum of $N^{\prime 2}$ electron-proton interaction potentials

$$
-e^{2} /\left|\boldsymbol{x}_{i}-\boldsymbol{y}_{\boldsymbol{j}}\right|
$$

$N^{\prime \prime}\left(N^{\prime \prime}-1\right) / 2$ electron-electron and as many proton-proton interactions

$$
+e^{2} /\left|\boldsymbol{x}_{i}-\boldsymbol{x}_{j}\right| \text { or }+e^{2} /\left(\boldsymbol{y}_{i}-\boldsymbol{y}_{j} \mid\right. \text {. }
$$


A useful trick for approximately calculating this, is to write $\lambda e^{2}$ instead of $e^{2}$ and to use

$$
G(\lambda=1)=G(\lambda=0)+\int_{0}^{1} \mathrm{~d} \lambda \frac{\mathrm{d} G(\lambda)}{\mathrm{d} \lambda} .
$$

In this way, we get the result

$$
Z^{\prime \prime}=\left(\frac{4 \pi^{2} m M}{\beta^{2} h^{4}}\right)^{z} V^{2 N^{\prime \prime}}\left(1-\frac{4 \pi N^{\prime \prime} e^{6}}{3 V A^{3}}\right)^{N^{\prime \prime}} \exp \left[-\beta \int_{0}^{1} \frac{\mathrm{d} \lambda}{\lambda} U(\lambda)\right],
$$

where $U(\lambda)$ is the internal energy by the interaction strength $\lambda e^{2}$, and can be written with the correlation functions $t_{+}$and $f_{-}$for particles of equal and unequal charges, respectively:

$$
U(\lambda)=\frac{N^{\prime \prime 2}}{V} \int_{\text {H.C. }} \mathrm{d} \boldsymbol{r} \frac{\lambda e^{2}}{r}\left[f_{1}\left(r, \lambda e^{2}\right)-f_{-}\left(r, \lambda e^{2}\right)\right] .
$$

We dropped terms of order $N^{\prime \prime}-1$. The correlation functions can be calculated with the B.B.G.K.Y. method, as has been done by Guernsey ${ }^{8}$ ) and Shure ${ }^{9}$ ). As a first approximation to this, we can take

$$
\begin{aligned}
& \left.f_{+}\left(r, \lambda e^{2}\right)=\exp \left[-\beta \lambda e^{2} / r\right]\right\} \text { for } r<e^{2} / A \\
& t_{-}\left(r, \lambda e^{2}\right)=0 \\
& f_{ \pm}\left(r, \lambda e^{2}\right)=1 \mp \frac{\beta \lambda e^{2}}{r} \mathrm{e}^{-\kappa \sqrt{ } \lambda r} \text { for } r>e^{2} / A
\end{aligned}
$$

with

$$
\kappa^{2}=\frac{8 \pi N^{\prime \prime} e^{2} \beta}{V} .
$$

With these approximations, we get the result

$$
\int_{0}^{1} \frac{\mathrm{d} \lambda}{\lambda} U(\lambda)=-\frac{2}{3} N^{\prime \prime} k e^{2}+\frac{4 \pi N^{\prime \prime} e^{6}}{2 V A^{2}}\left[1+A \beta-\frac{1}{3}(A \beta)^{2} \log (A \beta)+\ldots\right] .
$$

More refined expressions of the correlation functions only contain corrections in the small parameters

$$
\varepsilon_{1}=\frac{N^{\prime \prime} e^{6}}{V A^{3}} \sim\left[\frac{\left\langle r_{v}\right\rangle}{r_{0}}\right]^{3}
$$

and

$$
\varepsilon_{2}=\frac{\kappa e^{2}}{A}=2 \kappa\left\langle r_{p}\right\rangle
$$


(By saying that a function is of order $\varepsilon$, we shall mean hereafter that it does not contain larger terms than linear ones in both $\varepsilon_{1}$ and $\varepsilon_{2}$ ).

In order to have a good connection between our classical and quantummechanical description, we would then be obliged to carry also the quantummechanical calculations to a higher order in these parameters. This would mean:

1) to take account of the possibility of two or more electrons being at the same time in the immediate neighbourhood of the same proton;

2) to consider the screening effects of the plasma on the hydrogen energy levels.

There has been done some work on this last topic by Miss Harris 10), and the resulting corrections on the pressure are of the same order of magnitude as our corrections (obtained in section 5). We doubt, however, if her assumption of a Debye-screened potential is correct up to lowest bound states. In each case, this would lead us far out of the scope of our modest project, and we prefer not to do it. Although $\varepsilon_{1}$ and $\varepsilon_{2}$ can be of the same order of magnitude as $A \beta$, we take our approximations for $U$ to the second order in $A \beta$ and to the lowest order in $\varepsilon_{1}$ and $\varepsilon_{2}$. In that approximation the above result (10) is exact.

§3. Result. Putting (5), (6), (7) and (10) in (4), we have for the partition function of the plasma

$$
Z=\left[V\left(\frac{2 \pi M}{\beta h^{2}}\right)^{\mathrm{g}}\right]^{N} \sum_{N^{0}+N^{*}=N} \frac{N ! N !}{N^{0} ! N^{\prime \prime} ! N^{\prime \prime} !} g^{N^{0}} \cdot F^{N^{\prime \prime}},
$$

where we abbreviated for the following functions, after developing them to first order in $\varepsilon_{1}$ and $\varepsilon_{2}$,

$$
\begin{aligned}
F & =V\left(\frac{2 \pi m}{\beta h^{2}}\right)^{\sharp}\left\{1+\frac{2}{3} \beta \kappa e^{2}-\frac{\pi N^{\prime \prime} e^{6}}{V A^{3}}\left[\frac{4}{3}+2 A \beta+2(A \beta)^{2}-\right.\right. \\
& \left.\left.-\frac{2}{3}(A \beta)^{3} \log (A \beta)+\ldots\right]\right\}
\end{aligned}
$$

and

$$
\begin{aligned}
g & =\sum_{n=1}^{\nu} n^{2} \mathrm{e}^{-\beta E_{n}}+\left(\frac{2 \pi m}{\beta h^{2}}\right)^{\frac{4}{2}} \frac{\pi \mathrm{e}^{6}}{A^{3}}\left[\frac{4}{3}+2 A \beta-\frac{\sqrt{ } \pi}{3}(A \beta)^{\frac{1}{3}}+2(\mathrm{~A} \beta)^{2}-\right. \\
& \left.-\sqrt{ } \pi(A \beta)^{1}-\frac{2}{3}(A \beta)^{3} \log (A \beta)+\ldots\right] .
\end{aligned}
$$

We remark at once that the $N^{\prime \prime}$-dependent part of $F$ is much smaller than the independent part, and that $g$ is independent of $N^{0}$. There are two terms between the square brackets in the expression for $g$ that cancel out against 
analogous terms in the summation over the hydrogen levels, which can be calculated by partly substituting the summation by an integral, with

$$
E_{n}=-\frac{m e^{4}}{2 n^{2} \hbar^{2}} \text {. }
$$

In this way $g$ is of the form

$$
g=g^{\prime}+\left(\frac{2 \pi m}{\beta h^{2}}\right)^{\frac{\pi e^{6}}{A^{3}}}\left[\frac{4}{3}+2 A \beta+2(A \beta)^{2}-\frac{2}{3}(A \beta)^{3} \log (A \beta)+\ldots\right]
$$

where $g^{\prime}$ is independent of $A$.

On the partition function (11) we apply the usual variational techniques to get the largest term of the summation, and we drop the smaller terms. We must look for a maximum, both in $N^{0}$ and $N^{\prime \prime}$, of the expression

$$
\lambda\left(N^{0}+N^{\prime \prime}\right)+\log \left[\left(N^{0} ! N^{\prime \prime} ! N^{\prime \prime} !\right)^{-1} g^{N^{0}} F^{N^{\prime \prime}}\right],
$$

where $\lambda$ is a Lagrange multiplier, to be chosen afterwards so that $N^{0}+N^{\prime \prime}$ $=N$. Differentiation of this expression yields

$$
\begin{aligned}
& \lambda+\log g=\log N^{0} \\
& \lambda+\log F+\frac{N^{\prime \prime}}{F} \frac{\mathrm{d} F}{\mathrm{~d} N^{\prime \prime}}=\log N^{\prime \prime} .
\end{aligned}
$$

One immediately verifies that the last term of the left hand side of (16a) is of order $\varepsilon$. Consequently, we can rewrite this last equation as

$$
\lambda+\log f=\log N^{\prime \prime 2},
$$

where the relation between $f$ and $F$ can be written as

$$
f=F \cdot(1+s) \text { with } s=0(s) .
$$

The equations (15)-(16) have the solution

$$
N^{0}=a g ; N^{\prime \prime}=\sqrt{a f} .
$$

The constant $a=e^{\lambda}$ must be determined so that

$$
N=a g+\sqrt{a f}=a\left(g+\frac{f}{N^{\prime \prime}}\right) .
$$

Taking now only the largest term, and using (18), (19) and Stirling's formula we have

$$
\begin{aligned}
Z\left[V\left(\frac{2 \pi M}{\beta h^{2}}\right)^{!}\right]^{-N} \frac{1}{N ! N !} & =\frac{e^{a g}}{(a g)^{a,}} \cdot \frac{e^{2 \sqrt{a} f}}{(a f)^{\sqrt{a f}}} \cdot g^{a g} \cdot F^{\vee \overline{a f}}= \\
& =\frac{1}{N ! N^{\prime \prime} ! N^{\prime N-N^{\prime \prime}}}\left[\left(N^{\prime \prime} g+f\right) \cdot\left(\frac{F}{f}\right)^{N^{\prime \prime} / N}\right]^{N} .
\end{aligned}
$$


Using (17)-(19) one verifies directly that the square bracket can be written, to first order in $s$, as

$$
\left(N^{\prime \prime} g+F\right)^{N},
$$

where all terms containing $A \beta$ cancel out. The result is

$$
Z=\frac{N !}{N^{\prime \prime} ! N^{\prime \prime N-N^{\prime \prime}}}\left[V\left(\frac{2 \pi M}{\beta h^{2}}\right)^{\prime}\right]^{N}\left[N^{\prime \prime} g^{\prime}+V\left(\frac{2 \pi m}{\beta h^{2}}\right)^{*}\left(1+\frac{g}{3} \kappa e^{2} \beta\right)\right]^{N},
$$

which can easily be interpreted as the product of a statistical factor, a proton part and an electron part. As the proton and electron masses are not exactly equal to $M$ and $m$, it is perhaps better to write

$$
Z=\frac{N !}{N^{\prime \prime} ! N^{\prime \prime N-N^{\prime \prime}}}\left[N^{\prime \prime} V g^{\prime}\left(\frac{2 \pi M}{\beta h^{2}}\right)^{\prime}+V^{2}\left(\frac{4 \pi^{2} m_{e} m_{p}}{\beta^{2} h^{4}}\right)^{\prime}\left(1+\frac{2}{3} \beta e^{2} \kappa\right)\right]^{N} .
$$

The second part in the square brackets represents the contribution from the free particles, whereas the first one represents the atoms. The factor $N^{\prime \prime}$ in that first part can be understood as follows: if we add a new electron to the plasma, its probability to form an atom will be proportional to the number $N^{\prime \prime}$ of the free particles, with which it can form a bound state.

§4. Saha-equation and effective ionization potential. The ratio between the bound state part and the free particle part in (21) corresponds in a first approximation to the ratio between the numbers of neutral and ionized atoms in the plasma, which is nothing else than the well known Saha equation 11$)$. To obtain this equation with the corrections in $\varepsilon$, however, we cannot start from (21), but we must solve the exact equations (18). The corrections that appear can be interpreted as a lowering of the ionization potential. It is too obvious, however, that the corrections, which contain the arbitrary chosen $A$ explicitly, do not correspond to a physical effect on the ionization potential. They must rather be interpreted as arising from that part of our particles whose energy state is really in the neighbourhood of that corresponding to $A$. These particles can be thought as ionized or free, according to the choice of $A$. This must result in a dependence on $A$ of the ratio between the numbers of those two kinds of particle states. We are thus interested in an $A$-independent correction on the Saha equation, which we can find in the terms with $\beta e^{2} \kappa$. Retaining only those terms, we can calculate from (12) and (16) that

$$
f=V\left(\frac{2 \pi m}{\beta h^{2}}\right)^{\prime}\left(1+\beta e^{2} \kappa\right) .
$$


For $g^{\prime}$ we introduce a notation that corresponds more to the usual formulation of the Saha equation.

$$
g^{\prime}=\exp \left(-\beta E_{1}\right) \cdot Z_{B},
$$

where $E_{1}$ is the lowest hydrogen energy and $\left|E_{1}\right|$ the undisturbed ionization energy, and $Z_{B}$ is the partition function for the bound states of an atom, with the energies counted from the ground state. With these and (18), we have

$$
\frac{N^{\prime \prime}}{N^{0}}=\frac{V\left(\frac{2 \pi m}{\beta h^{2}}\right)^{\frac{3}{2}}\left(1+\beta e^{2} \kappa\right)}{N^{\prime \prime} \mathrm{e}^{\beta\left|E_{1}\right|} Z_{B}}
$$

or

$$
\frac{N^{\prime \prime 2}}{N^{0}}=\frac{V\left(\frac{2 \pi m}{\beta h^{2}}\right)^{z}}{e^{\beta\left(\left|E_{1}\right|-\kappa e^{2}\right)} Z_{B}}
$$

This result is in accordance with the calculations of Ecker and Kröll12) for the effective ionization potential in a low density plasma.

§5. Cut-independent expression for the partition function; pressure. Our result (20)-(21), though not explicitly dependent on $A$, still contains $N^{\prime \prime}$, which is a function of $A$. Until now, we always discerned between free and bound particles, although there is no sharp physical discrimination between them (a discussion of this fact has been given in reference 7). This way of looking is also pronounced in the obtained expressions for the partition function. It was our aim to derive results, independent of the definite choice of the ionization criterion, at least in the first order in $\varepsilon$. If this is true, it must be possible to obtain an expression for the partition function, not containing $A$ at all.

We first note that the statistical factor can be written, with Stirling's approximation formula, as

$$
\frac{N !}{N^{n} ! N^{n} N-N^{n}}=\left(\frac{\mathrm{e}^{n-1}}{n}\right)^{N},
$$

$n$ being defined as $N^{\prime \prime} / N$, the degree of ionization. This means that it indicates the fraction of electrons having energy above our arbitrary cut. An elementary calculation gives us

$$
n=\frac{f}{2 N g}\left[\sqrt{1+\frac{4 N g}{f}}-1\right] .
$$

The $A$-dependent parts of both $f$ and $N g$ are of order $\varepsilon$ in comparison with the $A$-independent part of $f$. The $A$-independent part of $\mathrm{Ng}$, which we called 
$N g^{\prime}$, can be either small or large, depending on temperature and density. In either case, however, we can obtain an approximate solution for (26) of the form

$$
n=n_{0}+q \text { with } \quad \frac{q}{n_{0}}=O(\varepsilon)
$$

and $n_{0}$ being the solution, which is provided by (26) after substitution of $g$ and $f$ by the $A$-independent expressions $g^{\prime}$ and

$$
F^{\prime}=V\left(\frac{2 \pi m}{\beta h^{2}}\right)^{\prime}\left(1+\frac{2}{3} \beta e^{2} \kappa\right) .
$$

This solution satisfies

$$
\frac{n_{0}^{2}}{1-n_{0}}=\frac{F^{\prime}}{N g^{\prime}}
$$

The exact meaning of this $n_{0}$ is not clear from its definition. It can perhaps be interpreted as a kind of effective degree of ionization, and it certainly does not differ much from the real degree of ionization, irrespective of the way in which one defines it. It depends on $A$ only through $\beta e^{2} \kappa$, which is of order $\varepsilon$. As the $A$-dependence of $\kappa$ is again an effect of order $\varepsilon$, the total $A$-dependence of $n_{0}$ is of the second order in $\varepsilon$, which we neglect.

We now calculate the $N$-th root of the product of the statistical and electronic part of the partition function (20):

$$
\begin{aligned}
& \frac{\mathrm{e}^{n-1}}{n}\left(N^{\prime \prime} g^{\prime}+F^{\prime}\right)=\mathrm{Ng}^{\prime} \mathrm{e}^{n_{0}-1} e^{q}\left(1+\frac{F^{\prime}}{N g^{\prime}\left(n_{0}+q\right)}\right)= \\
& =\frac{N g^{\prime} \mathrm{e}^{n_{0}-1}}{1-n_{0}}\left[1+O\left(q^{2}\right)\right] .
\end{aligned}
$$

It is worth noting that in the case of $n_{0} \approx 1$, this expression reduces to $F^{\prime} / n_{0}$. We thus derived the following expression for the partition function, independent of the cut up to the second order in $\varepsilon$ :

$$
Z^{1 / N}=V\left(\frac{2 \pi M}{\beta h^{2}}\right)^{\prime} \frac{N g^{\prime}}{1-n_{0}} \mathrm{e}^{n_{0}-1} .
$$

With this, we can derive an expression for the pressure, from the formula

$$
p=k T \frac{\mathrm{d}}{\mathrm{d} V} \log Z \text {. }
$$

This yields

$$
\frac{p}{k T N}=\frac{1}{V}+\frac{2-n_{0}}{1-n_{0}} \frac{\mathrm{d} n_{0}}{\mathrm{~d} V} .
$$


$n_{0}$ depends on the volume only through $F^{\prime}$. Derivation of (28) gives us

$$
\frac{\mathrm{d} n_{0}}{\mathrm{~d} V}=\frac{n_{0}\left(1-n_{0}\right)}{2-n_{0}} \frac{1}{F^{\prime}} \frac{\mathrm{d} F^{\prime}}{\mathrm{d} V},
$$

where $F^{\prime}$ depends on $V$ explicitly and implicitly through $\kappa$, which is a function of $n_{0}$. We can make an iteration of these derivatives in powers of $\varepsilon$, and in the first order the result is

$$
\frac{1}{F^{\prime}} \frac{\mathrm{d} F^{\prime}}{\mathrm{d} V}=\frac{1}{V}\left[1-\frac{\beta e^{2} \kappa}{3\left(2-n_{0}\right)}\right] .
$$

We then have for the pressure

$$
\frac{p V}{N k T}=\left(1+n_{0}\right)-n_{0} \frac{\beta e^{2} \kappa}{3\left(2-n_{0}\right)} .
$$

We could try to obtain a similar expression by adding the ideal gas result for the neutral particles, and the classical expression for the pressure of a plasma ${ }^{13}$ ) and we would get

$$
\frac{p V}{N k T}=\left(1-n_{0}\right)+2 n_{0}\left[1-\frac{\beta e^{2} \kappa}{6}\right] \text {. }
$$

With our method, we obtained a correction to the term containing $\beta e^{\mathbf{2}}$, which for $n_{0} \approx 0$ reduces to half its classically expected value. This correction can be understood from the fact that by compression of the volume the number of ionized particles will decrease by forming neutral bound states.

Acknowledgements. I should like to thank Professor N. G. van Kampen for his hospitality at the University of Utrecht, for suggesting the subject and for his helpful advice. I am also indebted to Professor L. P. Bouckaert and to Dr. D. Montgomery for their kind interest and stimulating discussions.

Reccived 30-3-65

\section{REFERENCES}

1) Herzfeld, K., Ann. Physik 51 (1916) 261.

2) Fowler, R. H., Phil. Mag. 45 (1923) 1.

3) Fermi, E., Z. Phys. 26 (1924) 54.

4) Becker, R., Z. Phys. 18 (1923) 325.

5) Planck, M., Ann. Physik 75 (1924) 673.

6) Riewe, K. H. and Rompe, R., Z. Phys. 111 (1938) 79.

7) Larenz, R. W., Comptes rendus de la VIe Conférence Internationale sur les Phénomènes d'Ionization dans les Gas, Paris (1963), Tome I, p. 219.

8) Guernsey, R. L., Phys. of Fluids 7 (1964) 792. 
9) Shure, F., Phys. Rev. Letters 12 (1964) 353.

10) Harris, G. M., Phys. Rev. 125 (1962) 1131 and $133 \mathbf{A}$ (1964) 427.

11) Cf. Thompson, W. B., "An introduction to plasma physics", Pergamon Press, Oxford (1962), p. 19 or Bates, D. R., "Atomic and molenular processes", Academic Press, New York (1962), p. 190 .

12) Ecker, G. and Kröll, W., Phys, of Fluids 6 (1963) 62.

13) Cf. Balescu, R., "Statistical Mechanics of charged Particles", Interscience Publishers, New York (1963), p. 249. 